\title{
Study on the Features of Flash Animation Videos of Coastal City
}

\author{
Yuan Lin \\ Hainan University, Haikou, Hainan, 570228 \\ hunter2011@foxmail.com
}

\begin{abstract}
Keywords: Unique Culture; Animated videos; Coastal City
\end{abstract}
\begin{abstract}
This paper outlines the concept of animated videos, characteristics, type and creation of truth at home and abroad, followed by the introduction of the status of regional characteristics and cultural development of animated videos and trends, and then focuses on the use of animated videos and other software development core technology, such as Photoshop, Flash, Maya (3ds Max ), Zbrush, After Effects (Premiere), TTPlayer, Gold Wave, For-mat Factory. The study on regional characteristics and cultural animation videos, the development of core technology to achieve the perfect fusion of technology and art, and high-quality animation videos has great importance for its geographical characteristics and cultural aspects, serve the local economic and social development, improve the fineness of green GDP, etc.
\end{abstract}

\section{Introduction}

The characteristics and culture not only provides a variety of themes for the animation design, animation and broaden the horizons of innovation make traditional commercials to promote its regional characteristics culture is mainly used camera and video post-synthesis technology creation, animation industry due to the rapid development of increasingly popular animated promo environment, the traditional video commercials cannot fully meet the visual and spiritual and cultural needs of the audience, therefore, study the regional characteristics of cultural propaganda movie sheet developed core technology, the development more attractive to the eye of the audience a variety of geographical features and cultural themes animation videos, for geographical features and cultural heritage of the Chinese nation, promote the construction of China's advanced culture, and promote animation culture industry development and promotion of green GDP couplers, etc., are It has a positive and realistic significance.

Animated videos, animated by definition is to play as the main form and content of the videos, it is especially emphasized the perfect blend of technical and artistic creation. A high quality animated video of the development process is more complicated, it has epidemic, high technology, multimedia content, artistic and other characteristics. The main types of animation videos include:(1) Type by animation is divided into: two-dimensional model, three-dimensional model, 2D and 3D animation videos superimposed type; (2) by way of propaganda into: Public Welfare animation videos (including PSAs, regional Featured cultural animation videos,) product/city/business/school/character animation videos and the like;(3) Press the animated character design style is divided into: impressionistic style, exaggerated comic style, lovely child style, decor and realistic style animated videos and other.

United States, South Korea and other world powers because of its successful animated cartoon commercial operation mode, the animation industry has reached the advanced level, every year to create a lot of high quality, widely influential animated videos, such as the US animation company Creation NBA animated propaganda tablets. Due to the late start of the animation, the animation creation level compared with developed countries, in a relatively backward level, but in recent years in China to develop policies to promote the animation industry, China's animation technology has improved significantly , higher quality domestic animation videos everywhere, and has been applied to various fields, value creation is considerable animation videos. 


\section{Geographical Characteristics, Cultural Status and Trend Analysis of the Development of the Animation Videos}

Development Status. Our geographical characteristics and culture generally refers to a long history in China within a particular region of the earth, unique and charm, heritage and today continues to play its role of traditional culture, which is unique in the region show folk, ecology, traditions of civilization. Our culture numerous geographical features, for example, only Suzhou City of Anhui on rich "Lingbi" "Yongqiao circus" "Sizhou play" "Dangshan" "Xiaoxian painting" "fuliji chicken" and other regional characteristics and culture. Geographical features and cultural animation videos is mainly used thematic animations commercials to promote its regional characteristics culture. In recent years, it became popular in the country, and effectively play its unique role. Now, in television, cinema, internet, bus car, train station and other places, it has been everywhere. Our animation studio, animation companies, film and television media companies and other units to develop a number of local characteristics and culture of anime videos, animated videos such as Taihu Lake in Wuxi, "Chinese pear township" (Dangshan pear) animated commercials, "Chinese circus village "animated videos, and so on. Generally speaking, China's geographical features and cultural development of animated commercials reality of the situation is excellent.

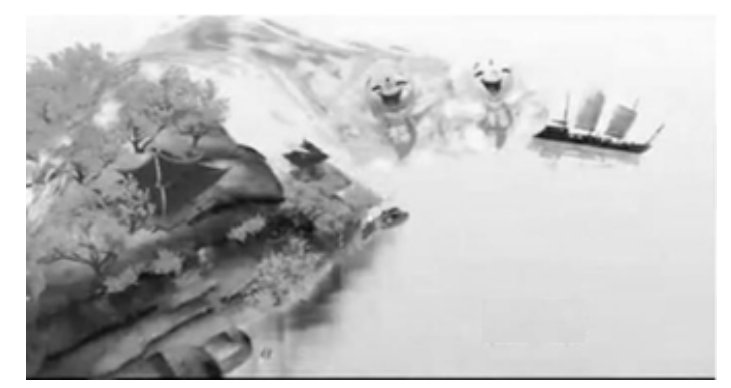

Fig. 1 The screenshots of Zhoushan animation promo

Trend Analysis. At present, the domestic regional characteristics and cultural animation videos developed gradually increased the number, quality and most creative commercials are high, can play a good publicity. But the domestic market there have been some low-quality videos developed products, animation videos to the application and promotion brought some negative impact. Low quality movie trailer is mainly due to the development of technology and artistry is not strong, but off, the domestic regional characteristics and cultural animation promo trend is toward the development of "high-quality" and "high efficiency" direction.

\section{The Core Technology of Animation Videos in Geographical Characteristics and Cultural Development}

Means and methods of regional characteristics and cultural development of a variety of animated videos, the core technology is two-dimensional, three-dimensional animation design and production, development software are mainly used Photoshop, Flash, Maya (or 3ds Max), Zbrush, After Effects (or Pre-miere), auxiliary tools have TT Player, Gold Wave, Format Factory and so on. An excellent animation videos in addition to local characteristics and culture should have emotional, rational, creative elements, should also achieve the perfect fusion of art and technology. In artistic terms of three requirements are: the role should be distinct characterization, plot to be appropriate, creative design; in the technical aspects of the three requirements are: high-tech, integrated and strong, a small amount of storage.

Technical Route Development. Geographical features and cultural animation videos developed technology roadmap points six steps: (1) collect geographical characteristics and cultural literature, fine note its cultural connotations; (2) In accordance with animation of early, middle, late three main stages, the main use of Photoshop, Flash, Maya (or 3ds max) and other software to create animated videos; (3) After Effects (or Premiere) software for its advanced special effects and post-synthesis; (4) use format Factory (format Factory) software to format conversion (converted to 
a smaller capacity FLV video formats); (5) the use of various media means to be publicity; (6) the development of their derivative products.

Software Production Technology. Flash-dimensional animation production process consists of pre, mid, post-production 3 process, specifically included script writing, picture storyboard, dialogue recording, the original painting design, art design and production (character image, props, color specified, scene design and production), animation, mixing sound recording, synthesis and release of the film and other production modules. (1) planning and script writing: determining regional characteristics and cultural animation promo piece long, animated main character and styling, write literary script. (2) Art and Design: Using Photoshop, Flash software, image design and scene design role, a major role to determine the color registration and scenes. (3) Sets of the storyboard screen: determine the number of sub-frame animated promo shots, draw each storyboard scene, writing screen content, determining the sub-mirror long, Scene, dialogue and music legend. (4) Lens design draft: draft draw a good lens design, fill out the picture size is set, the mirror number, duration and so on. (5) The animation design and production: design storyboard of the key action using Flash software, using frame by frame animation, room shape, motion, advanced animation (with ActionScript programming) production technology, one by one to create the original painting and intermediate Videos . (6) The scene graph and color: one by one lens drawn out scenes role of the active screen, and use Photoshop or Flash in the "Paint Bucket" tool storyboard scene color. (7) They were mixed recording and sound synthesis: the human voice, music, sound, according to art need for mixing the sound into Flash movies, make sound synthesis.

(8) Video synthesis output: Synthetic movie in Flash software, the final output video.

Maya 3D animation process can be divided into early, middle, three stages of post-production, it contains specific modeling, materials, maps, storyboard animation, lighting, 3D effects, layered rendering/synthesis, dub music, editing and other production module. (1) Modeling: including the establishment of animated scenes, animated props to establish and build a prototype of the character, which is the basis Maya animation. Maya modeling mainly polygonal modeling, patch modeling, surface modeling, polygon modeling is commonly used. (2) Materials, textures: the material is given to the model color, gloss and other effects. In accordance with a comprehensive proposal textures conceptual design and director, producer, etc., texture, color and texture and other 3D models for production. (3) Skinned: set its deformation and operation of the drive and other related parameters are required for 3D animation models (mostly role). (4) The storyboard animation: Reference literature screenplay and storyboard station present, according to the lens and time Layout for the role and activities of the object making the storyboard animation.

\section{Conclusion}

Develop a higher quality of geographical features and cultural animation videos, not only need for skilled application of software synthesis, but also need creative technology to integrate into the creation of art and innovative thinking. If only we achieve the perfect fusion of art and technology, can we fully reflect the value of the regional characteristics of cultural animation videos creation.

\section{References}

[1] Luo Nan. Flash Animation Videos "CG Gallery" Design and Implementation. Jinan: Shandong University, 2010: 1-2.

[2] Meng-Xiang G. Flash CS5 Animation Design Technology and Practice. Beijing: Tsinghua University Press, 2012: 7-10.

[3] Chen-Lu S. Maya Animation Technology Essence. Beijing: Tsinghua University Press, 2012: 23-26. 